\title{
ESTIMATING PHASE VELOCITY AND ATTENUATION OF GUIDED WAVES IN ACOUSTIC LOGGING DATA
}

\author{
by \\ K.J. Ellefsen and C.H. Cheng \\ Earth Resources Laboratory \\ Department of Earth, Atmospheric, and Planetary Sciences \\ Massachusetts Institute of Technology \\ Cambridge, MA 02139
}

K.M. Tubman

ARCO Oil and Gas Co. Plano, TX 75075

\begin{abstract}
Phase velocity and attenuation of guided waves have been estimated from acoustic logging data recorded by a receiving array. The method uses data from multiple sources and successive depths yielding more accurate estimates than could be obtained with data from one source and one depth. The inversion requires two steps: (1) all traces are transformed into the frequency domain with a fast Fourier transform, and (2) at each frequency a modification of Prony's method is used to estimate the guided waves' characteristics which include amplitude, attenuation coefficient, and wavenumber (which yields the phase velocity). An important assumption underlying this technique is that the formation, borehole fluid, and tool are homogeneous along the receiving array. Application of this method to synthetic data shows that the phase velocity and attenuation of the tube and pseudo-Rayleigh waves are accurately estimated at many frequencies. With noisy synthetic data, the phase velocities are correctly determined, but the attenuation estimates, being sensitive to noise, are accurate only when the amplitudes are high. Using data from multiple sources and successive depths suppresses the noise effects and improves both estimates. The amplitude estimates are important because they roughly indicate the reliability of the velocity and attenuation estimates. From laboratory and field data, the velocities for the guided waves are accurately predicted even when the amplitudes are low. The attenuation estimates are good when the amplitudes are high but degrade as the amplitudes diminish.
\end{abstract}




\section{INTRODUCTION}

In acoustic logging, the guided waves which propagate along the borehole are partly affected by the properties of the formation. Because some acoustic logging tools have many receivers, array processing methods can be used to calculate the phase velocity and attenuation of these waves. This information is crucial when estimating formation properties like permeability, which affects the tube wave's velocity and attenuation (Cheng et al., 1987; Williams et al., 1984; Staal and Robinson, 1977), anisotropy, which affects the guided waves' dispersion (White and Tongtaow, 1981), and S-wave velocity, which can be estimated from the tube wave's velocity in slow formations (Stevens and Day, 1986; Chen and Willen; 1984, Cheng and Toksöz, 1983).

The first array processing of acoustic logging data was done by Schoenberg et al. (1981), who tried to estimate the frequency-wavenumber spectrum. By using a Fourier transform over time with either another Fourier transform or the maximumlikelihood method over space, they were able to identify the tube and pseudo-Rayleigh waves in the two-dimensional spectrum. However, because the data consisted of only fourteen waveforms, the spectral estimates had poor resolution in the spatial dimension. To overcome this problem, Parks et al. (1983) and McClellan (1986) used a model based estimation technique, called the extended Prony's method, and obtained a high resolution spectrum from which accurate dispersion curves for the guided waves were calculated. Lang et al. (1987) implemented a variation of Prony's method which does not include the effects of attenuation and obtained seemingly accurate dispersion curves. A different technique, high-resolution slant stacking, has been used by Block et al. (1986) and Hsu and Baggeroer (1986) to obtain phase velocity estimates for the guided waves. This method, which requires extensive computations, yields phase velocity estimates but no attenuation or amplitude estimates.

The purpose of this paper is to present an improved method of estimating phase velocity and attenuation from acoustic logging data. By modifying Prony's method, data from successive depths and different sources on the same tool are combined to yield high quality estimates. Furthermore, the amplitudes are used to assess the accuracy of the estimates, especially the attenuation. The performance of this new method is demonstrated by processing synthetic, noisy synthetic, laboratory, and field data.

\section{METHOD}

As guided waves propagate along a borehole, the changes in their character are due to their phase velocity and attenuation. An example of multi-receiver data is displayed in Figure 1a, showing the traces which would be recorded by an acoustic logging tool. The increasing temporal duration of these guided waves is called dispersion and is due 
to changes in their phase velocity with frequency. The decrease in amplitude is due to intrinsic attenuation in the tool, fluid, and formation. Because geometric dispersion does not occur for guided waves, it does not affect the amplitude.

To accurately estimate the amplitude, attenuation, and phase velocity of the guided waves, a realistic mathematical model for the waves' behavior must be used. Lang et al. (1987) used contour integration in the complex wavenumber plane to demonstrate the appropriateness of their model. In contrast, the suitability of the model presented here will be argued heuristically although a mathematical proof virtually identical to that of Lang et al. (1987) is possible. Consider just the data at one frequency, say, $\omega_{j}$ (Figure 1b). Although these data samples in the frequency domain are complex numbers, their real parts may be sketched as shown in Figure 1c. At the first receiver, the wave's amplitude is $A\left(\omega_{j}\right)$, and its phase $\phi\left(\omega_{j}\right)$. As the wave propagates, its amplitude change is characterized by $\alpha\left(\omega_{j}\right)$, and its phase change by $k\left(\omega_{j}\right)$. The wave at some distance $z$ may be written as

$$
A\left(\omega_{j}\right) e^{\imath \phi\left(\omega_{j}\right)} e^{-\alpha\left(\omega_{j}\right) z} e^{\imath k\left(\omega_{j}\right) z}
$$

In this description, $\alpha\left(\omega_{j}\right)$ is the attenuation coefficient, which will be called just attenuation in this paper, and is related to the spatial quality factor, $Q_{s}$, via:

$$
\alpha\left(\omega_{j}\right)=\frac{\omega_{j}}{2 c\left(\omega_{j}\right) Q_{s}}
$$

where $c\left(\omega_{j}\right)$ is phase velocity (Aki and Richards, 1980).

The parameter $k\left(\omega_{j}\right)$ is the wavenumber, which describes the change in phase as the wave propagates along the borehole, and is related to the phase velocity via:

$$
c\left(\omega_{j}\right)=\frac{\omega_{j}}{k\left(\omega_{j}\right)} \text {. }
$$

Because the wave is recorded by receivers which only exist at discrete points, the expression for the wave in Eq. 1 must modified by replacing $z$ with $(n-1) \Delta z$ for which $n$ is an index varying from 1 to $N$, the total number of receivers, and $\Delta z$ is the receiver spacing. When more than one wave is present, a recorded sample, $x\left(n, \omega_{j}\right)$, may be expressed as a sum:

$$
\hat{x}\left(n, \omega_{j}\right)=\sum_{l=1}^{p} A_{l}\left(\omega_{j}\right) e^{\imath \phi_{l}\left(\omega_{j}\right)} e^{\left[-\alpha_{l}\left(\omega_{j}\right)+\imath k_{l}\left(\omega_{j}\right)\right](n-1) \Delta z}
$$

where $p$ equals the number of waves and is called the model order. The recorded sample is only approximated by $\hat{x}\left(n, \omega_{j}\right)$ since noise is always present.

The array processing involves changing the data from the time to the frequency domain by applying a fast Fourier transform to each trace and then estimating the 
parameters in Eq. 4 at every frequency. Because finding the parameters which minimize the squared error,

$$
\sum_{n=1}^{N}\left|x\left(n, \omega_{j}\right)-\hat{x}\left(n, \omega_{j}\right)\right|^{2}
$$

is a difficult, nonlinear problem, an approximate technique originally proposed by Gaspard Riche, Baron de Prony (1795) is used. Eq. 4 is written as

$$
\hat{x}(n)=\sum_{l=1}^{p} h_{l} z_{l}^{n-1}
$$

where the variable $\omega_{j}$ has been omitted to simplify the notation. The variables $h_{l}$ and $z_{l}$ are defined as

$$
h_{l}=A_{l}\left(\omega_{j}\right) e^{\imath \phi_{l}\left(\omega_{j}\right)}
$$

and

$$
z_{l}=e^{\left[-\alpha_{l}\left(\omega_{j}\right)+\imath k_{l}\left(\omega_{j}\right)\right] \Delta z}
$$

and are called the complex amplitude and exponent, respectively. With Prony's method, the complex exponents are estimated first using two equations for which the derivations are given in the appendix. A matrix equation is established with the data samples:

$$
\mathrm{Da}=-\mathbf{d}
$$

where

$\mathbf{D}=\left(\begin{array}{cccc}x(p) & x(p-1) & \ldots & x(1) \\ x(p+1) & x(p) & \cdots & x(2) \\ \vdots & \vdots & & \vdots \\ x(N-1) & x(N-2) & \cdots & x(N-p)\end{array}\right), \mathbf{a}=\left(\begin{array}{c}a(1) \\ a(2) \\ \vdots \\ a(p)\end{array}\right)$, and $\mathbf{d}=\left(\begin{array}{c}x(p+1) \\ x(p+2) \\ \vdots \\ x(N)\end{array}\right)$

This equation is commonly solved for a using the covariance method (Marple, 1987). The elements of $\mathbf{a}$ are the coefficients in the equation,

$$
z^{p}+a(1) z^{p-1}+\cdots+a(p-1) z+a(p)=0,
$$

for which the roots are the complex exponentials. Finding the roots is the nonlinear part of the estimation problem and is done with commonly available subroutines. Now that the exponents are known, Eq. 6 represents a linear relation between the complex amplitudes and the data samples. In matrix form, this equation is expressed as

$$
\mathbf{Z h}=\mathbf{x}
$$

where

$$
\mathbf{Z}=\left(\begin{array}{cccc}
1 & 1 & \cdots & 1 \\
z_{1} & z_{2} & \cdots & z_{p} \\
\vdots & \vdots & & \vdots \\
z_{1}^{N-1} & z_{2}^{N-1} & \cdots & z_{p}^{N-1}
\end{array}\right), \mathbf{h}=\left(\begin{array}{c}
h_{1} \\
h_{2} \\
\vdots \\
h_{p}
\end{array}\right) \text {, and } \mathbf{x}=\left(\begin{array}{c}
x(1) \\
x(2) \\
\vdots \\
x(N)
\end{array}\right)
$$


The method of least squares is used to solve this equation.

The accuracy of the phase velocity and attenuation estimates can be improved by exploiting the redundancy of information in acoustic logging data. The processing obtains average values for the phase velocity and attenuation of the guided waves over the length of the array. Between successive depths, the tool moves a small fraction of the array's length, and consequently the data sample nearly the same formation. In carefully chosen situations, data from several depths may be combined. Furthermore, for those logging tools having more than one source, the guided waves generated by each source sample the same formation, and these data may be combined. In this paper the data generated by each source at each depth will be called a data set.

The redundant information is included in the processing by modifying Prony's method. After, say, $M$ data sets have been transformed into the frequency domain, the matrix equation:

$$
\left(\begin{array}{c}
\mathbf{D}_{1} \\
\mathbf{D}_{2} \\
\vdots \\
\mathbf{D}_{M}
\end{array}\right) \mathbf{a}=-\left(\begin{array}{c}
\mathbf{d}_{1} \\
\mathbf{d}_{2} \\
\vdots \\
\mathbf{d}_{M}
\end{array}\right)
$$

is used to find the vector a at each frequency. Submatrix $\mathbf{D}_{i}$ and subvector $\mathbf{d}_{i}$ come from one data set and are identical to $\mathbf{D}$ and $\mathbf{d}$ in Eq. 10. Because each submatrix and subvector are independent of the others, data sets from sources having completely different characteristics can be combined, improving the estimates of a and ultimately of the velocity and attenuation. Eq. 14 is solved by the method of least squares, and then the roots of Eq. 11 yield the complex exponents. The complex amplitudes are calculated using Eq. 12 with only one data set because the amplitudes may differ for each source.

In summary, processing of acoustic logging data requires two operations. (1) All data sets are transformed into the frequency domain by applying a fast Fourier transform to each trace. (2) For each frequency, the data sets are combined to estimate $z_{l}$ and $h_{l}$ by a modification of Prony's method, which requires applying Eqs. 14, 11, and 12 in this order. The complex exponential determines attenuation and wavenumber (Eq. 8), and complex amplitude the real amplitude (Eq. 7). Wavenumber is converted to phase velocity using Eq. 3. Although the phase is determined by this estimation procedure, this parameter would not be used in any inversion for formation properties and will not be discussed again.

An important assumption is that the formation, borehole fluid, and tool are homogeneous along the receiving array. Because reasonable estimates are obtained from field data, this assumption is believed to be adequately satisfied for most situations. When this assumption is seriously violated, which would occur, for example, at a boundary between very different formations or at a washout, the parameter estimates will not 
be accurate.

The quality of the phase velocity and attenuation estimates is degraded by noise, and several techniques may be used to diminish its effects. Combining several data sets improves the accuracy of the parameter estimates, and this technique is demonstrated in the Results section. Another method is to increase $p$ in Eq. 4 until it is equal to or is slightly less than half the number of receivers, $N / 2$. The extra parameters model the noise and thereby increase the accuracy of those parameters modeling the actual waves. Another approach, which was originally proposed by McClellan (1986), is to process only the data samples associated with the desired wave. This selection is easily accomplished by applying a window in time to each trace. The window keeps the desired wave and sets the remainder of the trace to zero. Standard processing yields the parameter estimates for the wave, but to limit the computational burden the processing is only performed for those frequencies at which the wave is expected to exist and have a reasonably large amplitude. When processing field data, all of these techniques are usually used.

\section{RESULTS}

\section{Synthetic Data}

Noiseless synthetic data were processed to determine the inversion's performance under controlled conditions. The first test used data from one source and one depth (Figure 1a). For processing, the model order was selected to be six, half the number of receivers, and the waveforms were not windowed. All synthetic data presented here were processed in this manner. The amplitude, phase velocity, and attenuation estimates are shown in Figures $2 a, b$, and $c$, respectively. The processing detected a low amplitude tube wave which is not easily observed in the traces. At all frequencies the phase velocity estimates are very close to the correct values. Although the attenuation estimates are reasonably accurate, their slight scatter suggests that they are somewhat less robust than the other estimates. Attenuation plotted as $1 / Q$ (Figure 3 ) is much more scattered than the attenuation coefficient estimates because two estimates (i.e., wavenumber and attenuation coefficient) are needed to compute $1 / Q$. Because the attenuation coefficient is the parameter which is estimated and would be used in an inversion for formation properties, only it will be displayed.

When few traces are available, the inversion has difficulty determining the parameter estimates for two or more waves at the same frequency. For example, the estimates for the low amplitude tube wave end at about $9 \mathrm{kHz}$ (Figure 2), although the wave is known to exist at higher frequencies. Above this threshold, the algorithm, using the twelve samples corresponding to the twelve receivers, yields slightly biased tube wave 
estimates due to the presence of the high amplitude pseudo-Rayleigh wave. Experience has shown that the estimates for the higher amplitude wave are fairly accurate.

The effect of noise upon the parameter estimates was determined by processing synthetic data to which Gaussian random noise was added. The last trace from the original data set (Figure 2a) is compared to the last trace from the noisy data set in Figure 4. Noise of this magnitude is generally larger than is found in field data and served as a good test of the inversion's robustness. The processing results are shown in Figures $2 \mathrm{~d}$, e, and $f$. The amplitude and phase velocity estimates are very similar to those from the noiseless data indicating that these estimates are quite robust. The attenuation estimates for the high amplitude pseudo-Rayleigh wave are moderately accurate whereas those for the low amplitude tube wave are scattered. The accuracy of these estimates largely depends upon the signal to noise ratio.

To improve the parameter estimates from noisy synthetic data at one depth, noisy data from three depths were combined. Each data set was generated by adding Gaussian random noise like that shown in Figure 4 to the traces displayed in Figure 1a. The processing results are shown in Figures $2 \mathrm{~g}, \mathrm{~h}$, and $\mathrm{i}$. The amplitude and phase velocity estimates are smoother than those for one depth and closely resemble the estimates from the noiseless data. The attenuation estimates are markedly closer to the correct values.

Combining data sets from two sources improved the accuracy of the parameter estimates. Both data sets (Figures 1a and 5) cannot be simply combined to form one set with twenty-four traces because their sources are different and the distance between the sources is less than the array length. However this problem is easily overcome because Eq. 14 treats the data sets individually. The processing results are shown in Figures $2 \mathrm{j}, \mathrm{k}$, and $\mathrm{l}$. Using data from the additional source generally yielded slightly better phase velocity and attenuation estimates than were obtained with data from only one source (Figures $2 b$ and $c$ ). The tube wave's attenuation estimates at high frequencies are slightly biased because the pseudo-Rayleigh wave has a large amplitude. When processing field data which may contain noise, the additional source would probably improve these estimates noticeably.

\section{Laboratory Data}

Processing laboratory data was an ideal test for the method because the fluid and formation properties are well known and a tool, which would complicate the wave field, was not present. The data (Figure 6) were collected in a water-filled borehole through a lucite block. Because the lucite's S-wave velocity is less than the fluid's Pwave velocity, the leaky- $P$ wave is prominent, and the pseudo-Rayleigh wave does not exist. In addition to the tube wave, the processing detected five modes of the leaky-P 
wave (Figure 7). The phase velocity estimates are very accurate even when several modes exist at the same frequency because forty-three samples - corresponding to the forty-three traces - are available to estimate the modes' parameters. At high frequencies, the third and fourth leaky-P modes having somewhat low amplitudes are slightly biased. The attenuation estimates are accurate when the amplitude is high which occurs for the tube wave, for the leaky-P modes near their cutoff frequency, and also for first leaky-P mode above $300 \mathrm{kHz}$. In general the quality of the attenuation estimates degrades as the amplitudes diminish.

An interesting feature is the large amplitude event in the leaky- $P$ wave which is easily seen in those traces which are far from the source (Figure 6). Some researchers have called similar events in field data fluid waves because their moveout nearly equals the fluid's $P$-wave velocity. However, the processing shows that this event is the high frequency portion of the first leaky- $\mathrm{P}$ mode which has a large amplitude and a phase velocity virtually equal to the fluid's $\mathrm{P}$-wave velocity. These data substantiate the numerical studies on leaky modes conducted by Paillet and Cheng (1986).

\section{Field Data}

The processing results from the field data demonstrate the inversion's performance under realistic conditions and, furthermore, show the effects of permeability upon velocity dispersion. Data consisting of ten traces (Figure 8) were collected in a sandstone formation in which the permeability generally ranges from 200 to $300 \mathrm{mD}$ and occasionally reaches 600 to $700 \mathrm{mD}$. The field logs indicate that the borehole wall is smooth and that the density is fairly constant. The estimates for the amplitude, phase velocity, and attenuation are shown in Figures $9 \mathrm{a}, \mathrm{b}$, and $\mathrm{c}$, respectively, and were obtained by combining data sets from four depths. The complicated behavior of the amplitude estimates is due to the source's frequency spectrum. The phase velocity estimates are believed to be reasonably accurate at all frequencies. The attenuation estimates for the tube wave are probably most accurate at the low frequencies where only one wave is present and seem to degrade near $6 \mathrm{kHz}$ at which the pseudo-Rayleigh wave begins. Between 6 and $10 \mathrm{kHz}$ where the pseudo-Rayleigh wave has a relatively large amplitude, the attenuation estimates seem reasonable. Beyond $10 \mathrm{kHz}$ the estimates are scattered because the amplitude is quite low. The phase velocity and attenuation predictions, which were based on the Biot-Rosenbaum model (Cheng et al., 1987) and were constrained by measurements of the saturated $\mathrm{P}$ - and S-wave velocities, borehole fluid density, formation density, and borehole radius, match the data reasonably well. In particular, the low velocity of the low frequency tube wave - a characteristic of highly permeable formations - is fairly well modeled. 


\section{DISCUSSION}

The results of the noise tests show that reliable parameter estimates can be obtained from low quality synthetic data, but this conclusion does not always apply to field data. The processing, which was performed on a VAX 11-780 computer for the results presented in this paper, was also tested on a lower precision IBM 4381 computer. With the IBM machine, the phase velocity estimates were generally biased about one percent. The attenuation estimates varied much more about their theoretical predictions but did not seem biased. Based upon this experience, the processing results from field data, for which the precision depends upon the digitization, may also show similar effects. Another important consideration is the effect of nonrandom noise which might come from formation heterogeneity, mismatched receivers, or electronic problems like clipping. The experience gained from processing field data indicates that these problems can be ameliorated by carefully examining the acoustic logging data and other borehole measurements like the caliper log before processing. Because these problems appear to be infrequent, most of the data can probably be processed to obtain reasonable phase velocity and attenuation estimates.

The processing time is quite short. In general, as the model order or the number of data sets increases, the processing time increases. Excluding input and output operations, about six to eight cpu seconds on the IBM 4381 computer were needed to calculate the parameter estimates.

Before this method can be routinely applied to logging data, an automated method to separate the parameter estimates for the actual waves from those for the noise must be developed. Almost always a large model order is selected to model the noise and thereby improve the accuracy of the estimates for the waves. Many of these extraneous estimates can be eliminated if their velocity or attenuation estimates fall outside reasonable ranges. For example, the attenuation must be positive and less than some upper bound, which depends upon the formation and borehole fluid conditions. Lang et al. (1987) eliminated the remaining, extra estimates when their amplitude fell below an established threshold, but using their approach low amplitude waves, which could have accurate phase velocity estimates, would be removed. For this paper, the extra estimates were deleted manually.

The processing results for the synthetic, laboratory, and field data show that the higher the amplitude the greater the reliability of the phase velocity and particularly the attenuation estimates. When performing an inversion for formation properties, the amplitude information can be used to weigh the data, which should improve the inversion's performance. 


\section{CONCLUSIONS}

Array processing methods may be used to estimate phase velocity and attenuation for the guided waves over a broad range of frequencies. The technique presented here combines a fast Fourier transform over time with Prony's method over space. This method is based upon a model which is appropriate for the guided waves and from which the amplitude, attenuation, and phase velocity may be estimated at each frequency. The accuracy of these estimates may be improved by combining data from multiple sources and successive depths. The assumption underlying this processing method, that the formation, borehole fluid, and tool are homogeneous along the receiving array, appears to be adequately satisfied for most field situations.

This array processing method was applied to synthetic data to determine its performance. Generally the phase velocity estimates are very accurate even when the waves have low amplitude or when the data are noisy. Attenuation estimates are somewhat sensitive to noise, and accurate estimates can only be obtained when the signal to noise ratio is high. Prony's method has difficulty estimating the parameters for two or more waves at the same frequency when the array has only a few receivers (e.g., about twelve). For this situation, the phase velocity and attenuation estimates for the highest amplitude wave are fairly accurate, but those for the lower amplitude waves should be ignored. Including data from multiple sources and depths improves the phase velocity and attenuation estimates.

The tests with the laboratory and field data corroborate what was learned with the synthetic data. The velocity estimates are accurate over a broad range of frequencies even when the amplitude is low. The attenuation estimates are reliable when the amplitude is high but become inaccurate as the amplitude diminishes. The velocity and attenuation estimates will be useful when performing an inversion for formation properties.

\section{ACKNOWLEDGEMENTS}

We wish to thank Gregory L. Duckworth for his helpful thoughts and suggestions concerning this project. The laboratory data were provided by Fatih Guler. Jim Watson and Tom Shoberg from the ARCO Oil and Gas Company helped us program ARCO's

computer. Karl J. Ellefsen and C. H. Cheng are supported by the Full Waveform Acoustic Logging Consortium at M.I.T. 


\section{REFERENCES}

Aki, K., and Richards, P. G., Quantitative Seismology, W. H. Freeman \& Co., 1980.

Block, L. V., Cheng, C. H., and Duckworth, G. L., Velocity analysis of multi-receiver full waveform acoustic logging data in open and cased holes: Presented at the 56th Ann. Internat. Mtg., Soc. Explor. Geophys., Expanded Abstracts, 33-35, 1986.

Chen, S. T., and Willen, D. E., Shear wave logging in slow formations, Trans. SPWLA 25th Ann. Logging Symp., paper DD, 1984.

Cheng, C. H., and Toksöz, M. N. , Determination of shear wave velocities in slow formations, Trans. SPWLA 24th Ann. Logging Symp., paper V, 1983.

Cheng, C. H., Zhang, J., and Burns, D. R., 1987, Effects of in-situ permeability on the propagation of Stoneley (tube) waves in a borehole, Geophysics, 52, 1279-1289, 1987.

de Prony, Baron (Gaspard Riche), Essai expérimental et analytique: sur les lois de la dilatabilité de fluides élastiques et sur celles de la force expansive de la vapeur de l'eau et de la vapeur de l'alkool, à différentes températures: J. E. Pollytech., 1, 24-76, 1795.

Hsu, K., and Baggeroer, A. B., Application of the maximum-likelihood method (MLM) for sonic velocity logging, Geophysics, 51, 780-787, 1986.

Lang, S. W., Kurkjian, A. L., McClellan, J. H., Morris, C. F., and Parks, T. W., Estimating slowness dispersion from arrays of sonic logging data, Geophysics, 52, 530-544, 1987.

Marple, S. L., Digital Spectral Analysis with Applications, Prentice-Hall, Inc., 1987.

McClellan, J. H., Two-dimensional spectrum analysis in sonic logging, Acoust., Speech, and Sig. Proc. Mag., July, 12-18, 1986.

Paillet, F. L., and Cheng, C. H., A numerical investigation of head waves and leaky modes in fluid-filled boreholes, Geophysics, 51, 1438-1449, 1986.

Parks, T. W., McCellan, J. H., and Morris, C. F., Algorithms for full-waveform sonic logging, Proc. 2nd Acous, Speech, and Sig. Proc. Workshop on Spec. Est., 186-191, 1983.

Schoenberg, M., Marzetta, T., Aron, J., and Porter, R. P., 1981, Space-time dependence of acoustic waves in a borehole, J. Acoust. Soc. Am., 70, 1496-1507, 1981. 
Staal, J. J., and Robinson, J. D., Permeability profiles from acoustic logging data, Presented at 52d Ann. Fall Tech. Conf., Soc. Petr. Eng., Am. Inst. of Min., Metall. Petr. Eng., 1977.

Stevens, J. L., Day, S. M., Shear velocity logging in slow formations using the Stoneley Wave, Geophysics, 51, 137-147, 1986.

White, J. E. and Tongtaow, C., Cylindrical waves in transversely isotropic media, $J$. Acoust. Soc. Am., 70, 1147-1155, 1981.

Williams, D. M., Zemanek, J., Angona, F. A., Dennis, C. L., Caldwell, R. L., The long spaced acoustic logging tool, Trans. SPWLA 25th Ann. Logging Symp., paper T, 1984. 


\section{APPENDIX}

The derivation of the equations needed to calculate the complex exponentials will be presented in this appendix. Although similar material has been published (e.g. Marple, 1987), the derivation is included to make the paper complete. The starting point for the derivation is the equation,

$$
\hat{x}(n)=\sum_{k=1}^{p} h_{k} z_{k}^{n-1},
$$

for which the variables are defined in the main body of the paper. Prony (1795) recognized that this equation is the solution to some homogeneous, linear, constantcoefficient, difference equation which could be used to find the complex exponentials. To find this difference equation, a polynomial, $\phi(z)$, having the exponents for its roots, is defined as

$$
\phi(z)=\prod_{k=1}^{p}\left(z-z_{k}\right) .
$$

After performing the multiplication, $\phi(z)$ may be expressed as a summation:

$$
\phi(z)=\sum_{m=0}^{p} a(m) z^{p-m}
$$

where $a(m)$ are complex coefficients with $a(0)=1$.

Eq. A-1 and A-3 will now be used to determine the difference equation. Eq. A-1 has its index shifted by $-m$, is multiplied by $a(m)$, and is summed over the index $m$ to give:

$$
\begin{aligned}
\sum_{m=0}^{p} a(m) \hat{x}(n-m) & =\sum_{k=0}^{p} h_{k} \sum_{m=0}^{p} a(m) z_{k}^{n-m-1} \\
& =\sum_{k=0}^{p} h_{k} z_{k}^{n-1-p} \sum_{m=0}^{p} a(m) z_{k}^{p-m}
\end{aligned}
$$

which is valid for $p+1 \leq n \leq 2 p$. The second summation on the right hand side is $\phi(z)$ evaluated at a root, $z_{k}$, and consequently is zero. The left hand side may be rewritten as the difference equation:

$$
\hat{x}(n)=-\sum_{m=1}^{p} a(m) \hat{x}(n-m)
$$

which appears in matrix form as $\mathbf{D a}=-\mathbf{d}$ within the main body of the paper. After this equation is solved for the coefficients, $a(m)$, the roots of the characteristic equation, (i.e., Eq. A-3),

$$
z^{p}+a(1) z^{p-1}+\cdots+a(p-1) z+a(p)=0,
$$

yield the complex exponents. 


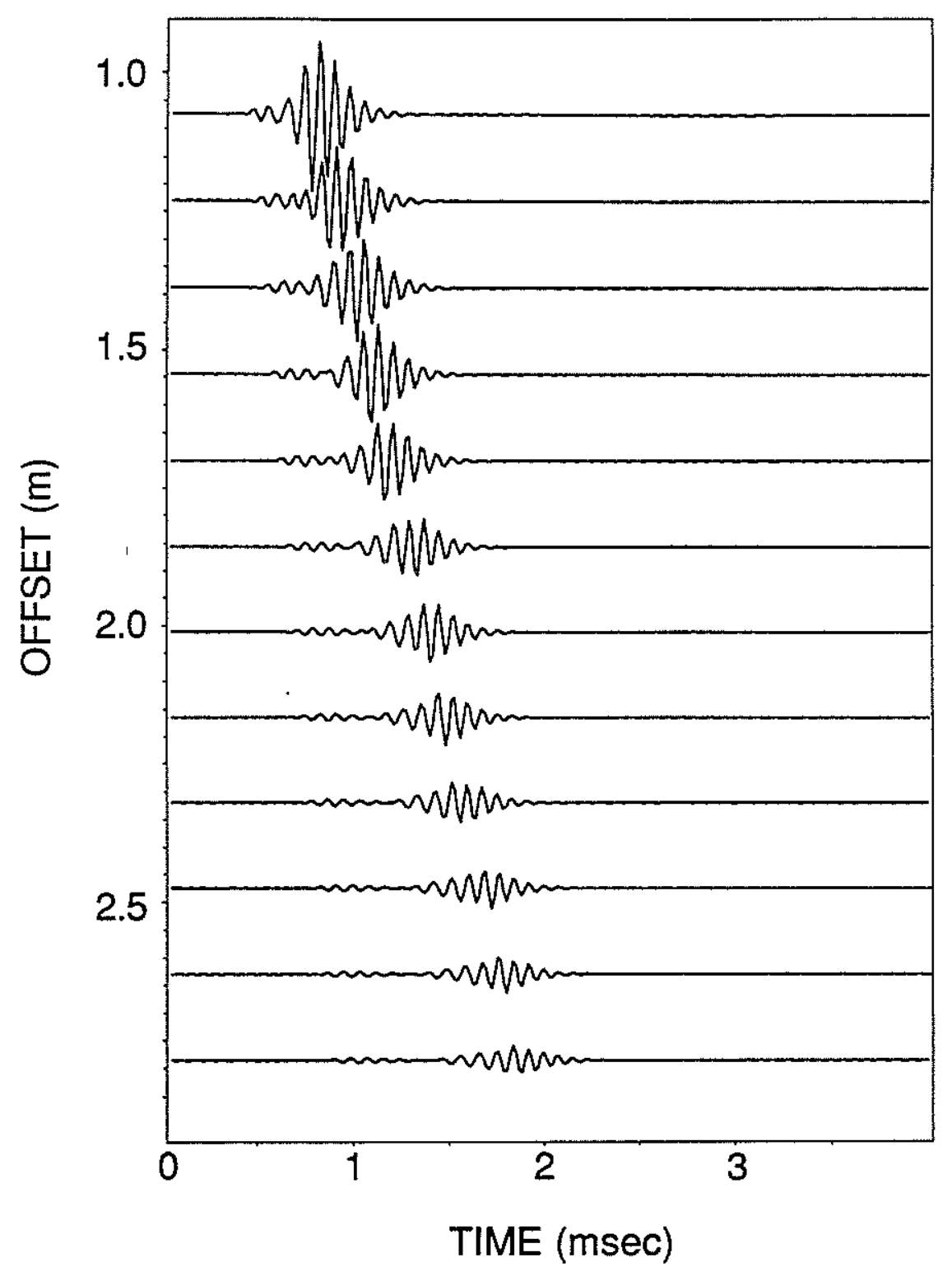

Figure 1: Processing multi-receiver acoustic logging data. (a) The traces record the wave field as it passes the acoustic logging tool. This data set was used to test the processing method. (b) The first processing step is convert the data to the frequency domain by applying a fast Fourier transform to each trace. These graphs show the magnitude of the frequency spectrum. (c) The second step is to estimate the guided waves' properties at each frequency. The samples at one frequency, shown by the black squares in part (b), are complex, but their real parts describe the wave's amplitude, $A$, phase, $\phi$, attenuation, $\alpha$, and wavenumber, $k$, which are estimated by Prony's method. 


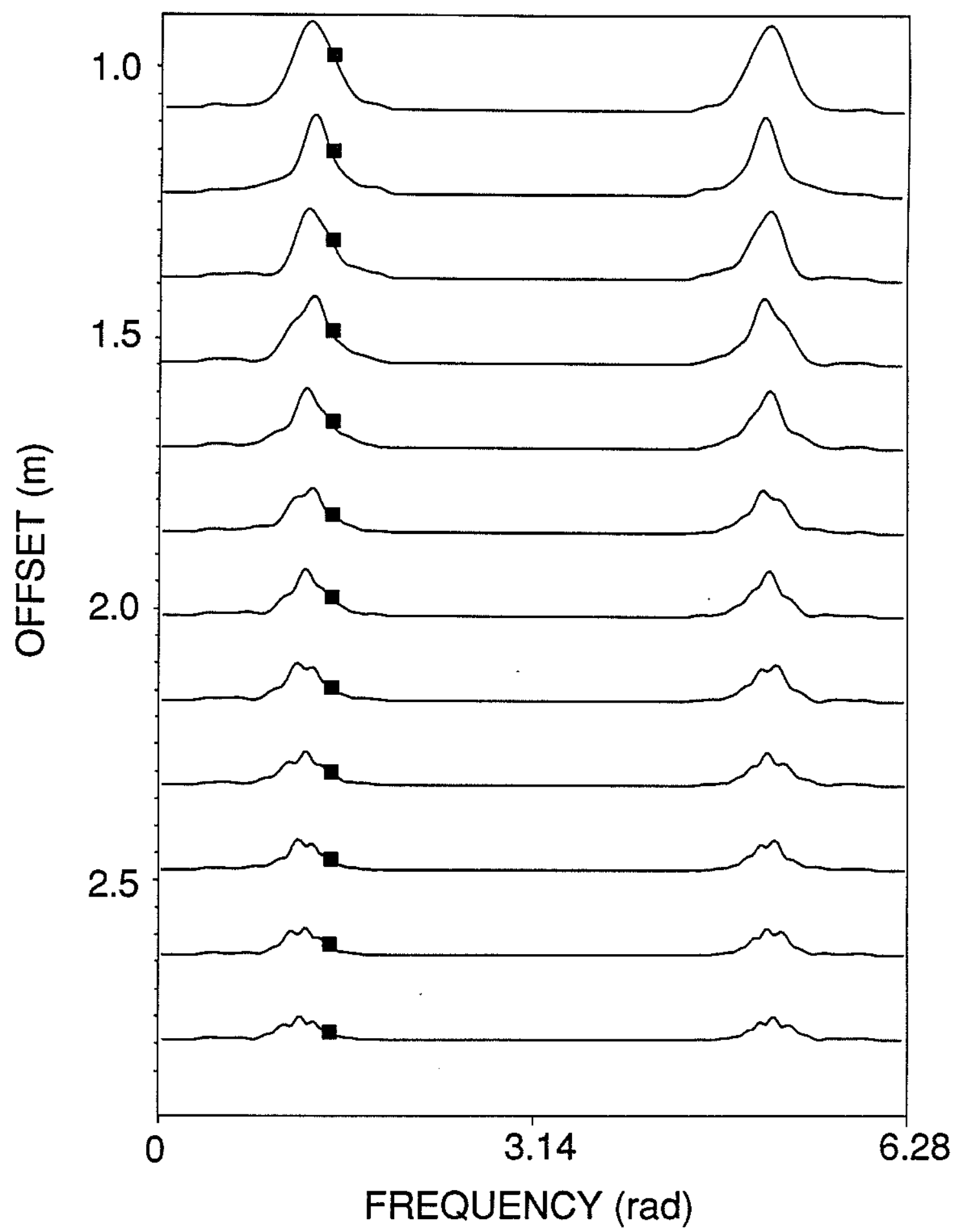




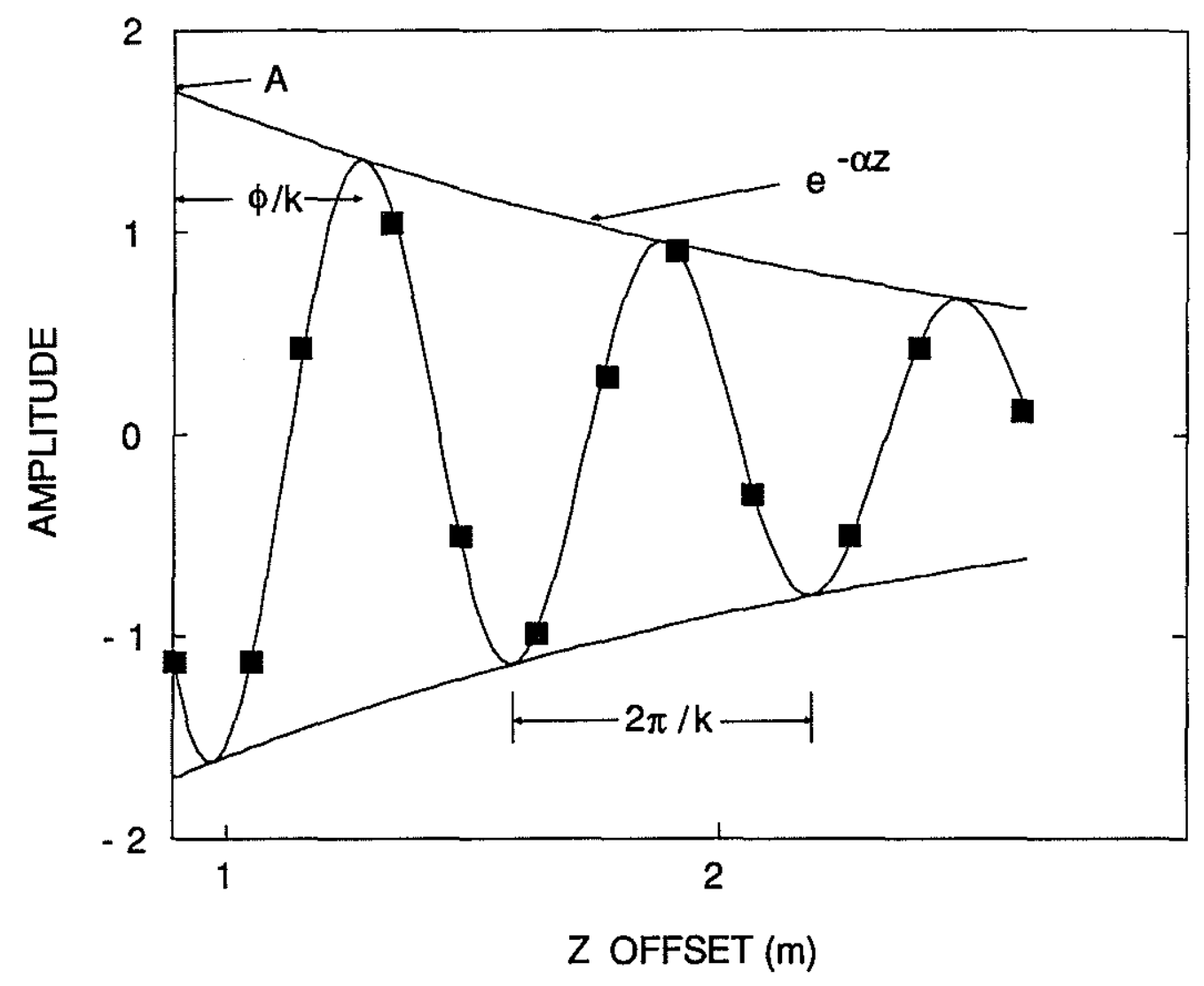



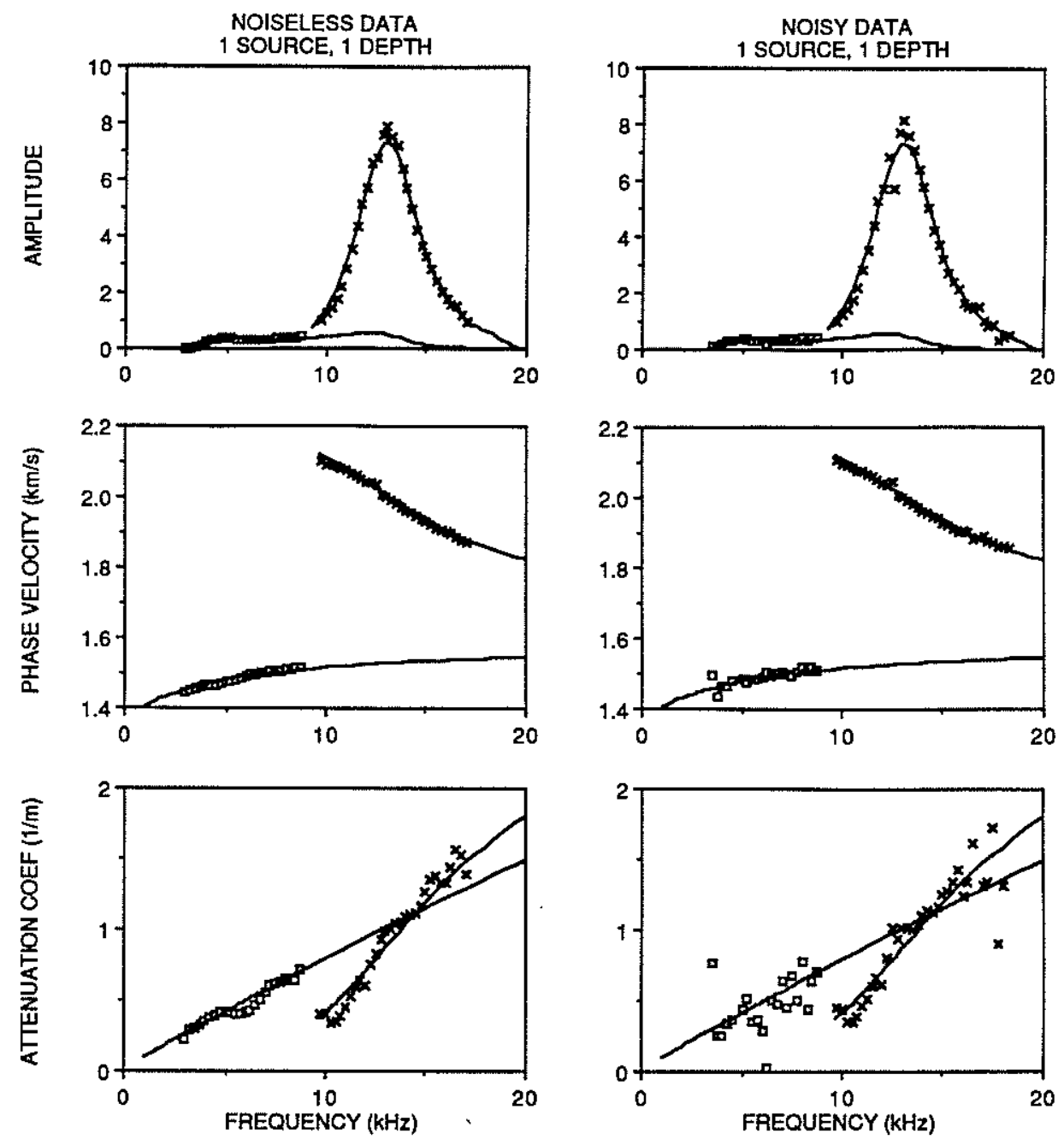

Figure 2: Amplitude, phase velocity, and attenuation estimates from the synthetic data. In this and all subsequent figures, an estimate for the tube wave is shown by an open square, and for the pseudo-Rayleigh wave by an " $x$ ". The solid lines are the correct values. The first three tests used the data shown in Figure 1a. (a), (b), and (c) Results for noiseless data from one source and one depth. (d), (e), and (f) Results for noisy data from one source and one depth. Random Gaussian noise was added to the traces, and the last trace before and after contamination is shown in Figure 4. (g), (h), and (i) Results for noisy data from one source and three depths. The noise level was identical to that in the previous example. (j), (k), and (l) Results for noiseless data from two sources and one depth. This test used the data sets shown in Figures $1 \mathrm{a}$ and 5 , and the amplitudes were estimated for the latter set. 
Ellefsen et al.
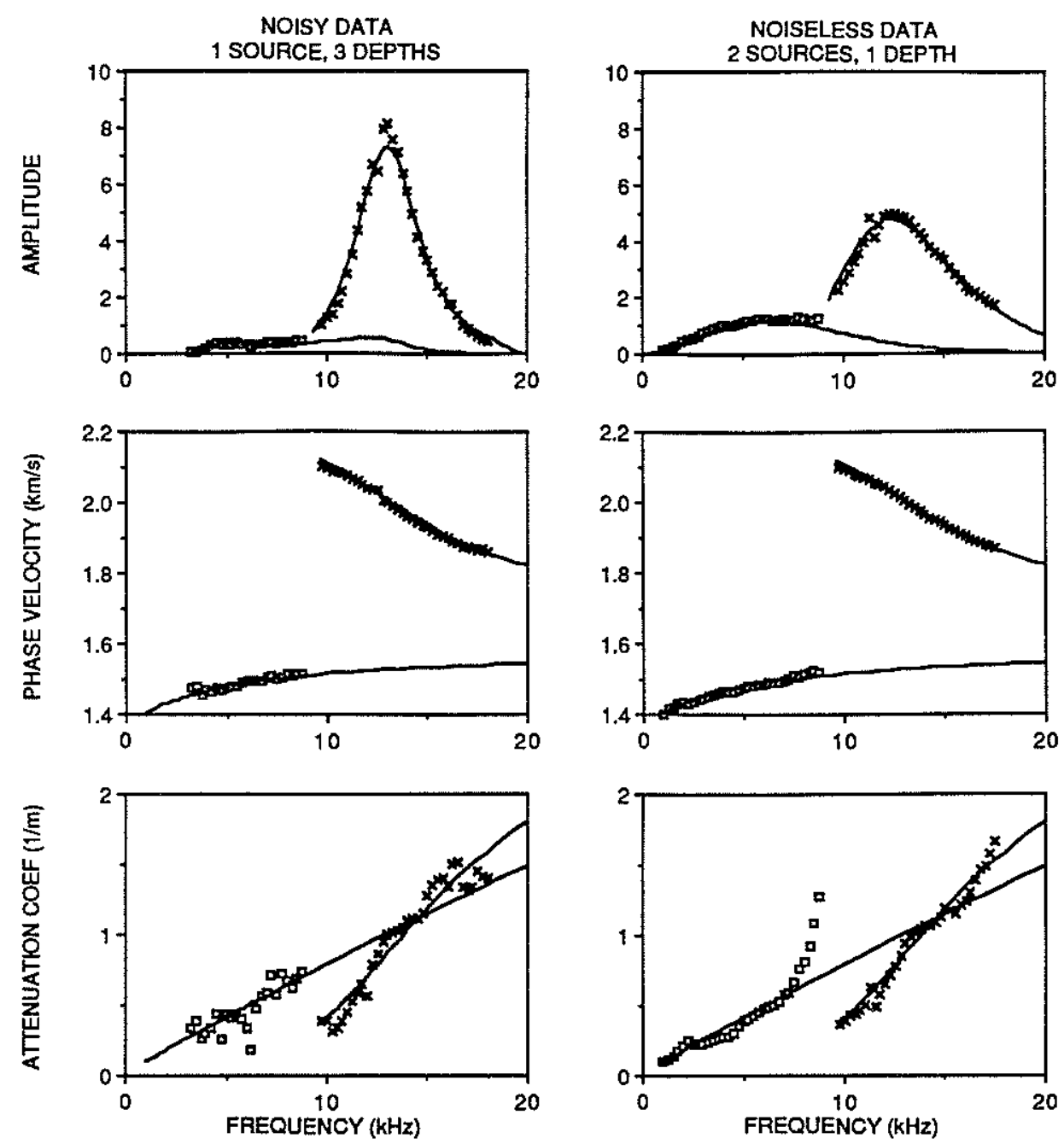


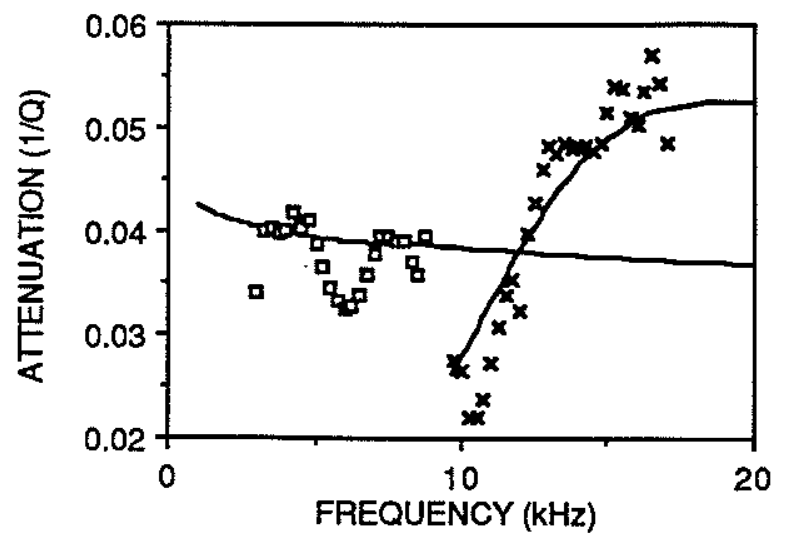

Figure 3: Attenuation plotted as $1 / Q$ for the noiseless synthetic data with one depth and one source. 


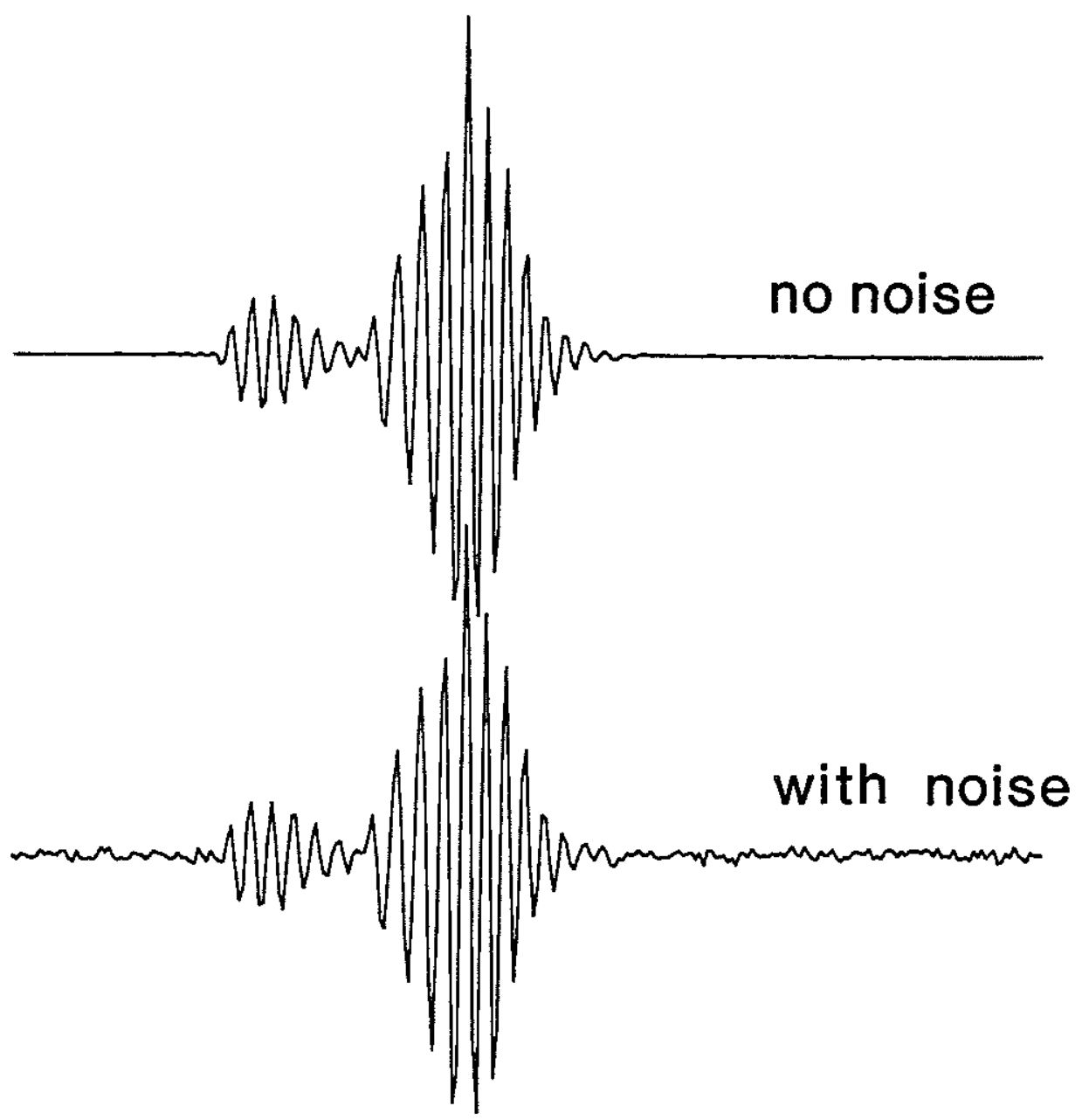

Figure 4: Last trace of the synthetic data (Figure 1a) before and after Gaussian random noise was added. Noise having a similar magnitude was added to all traces. 


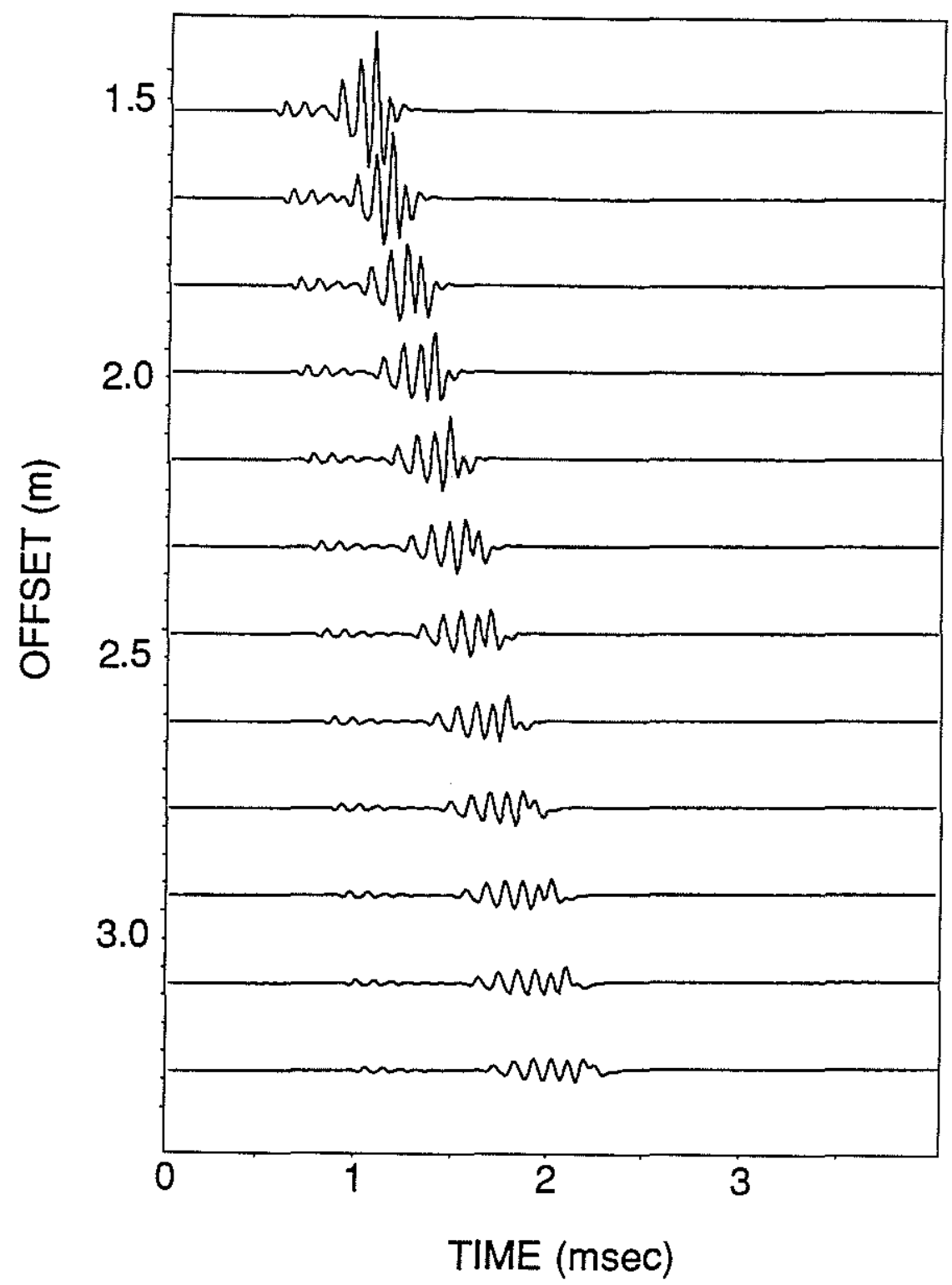

Figure 5: Synthetic data set which was combined with that shown in Figure 1a. The character of the waves in the two data sets differs because the sources are different. 


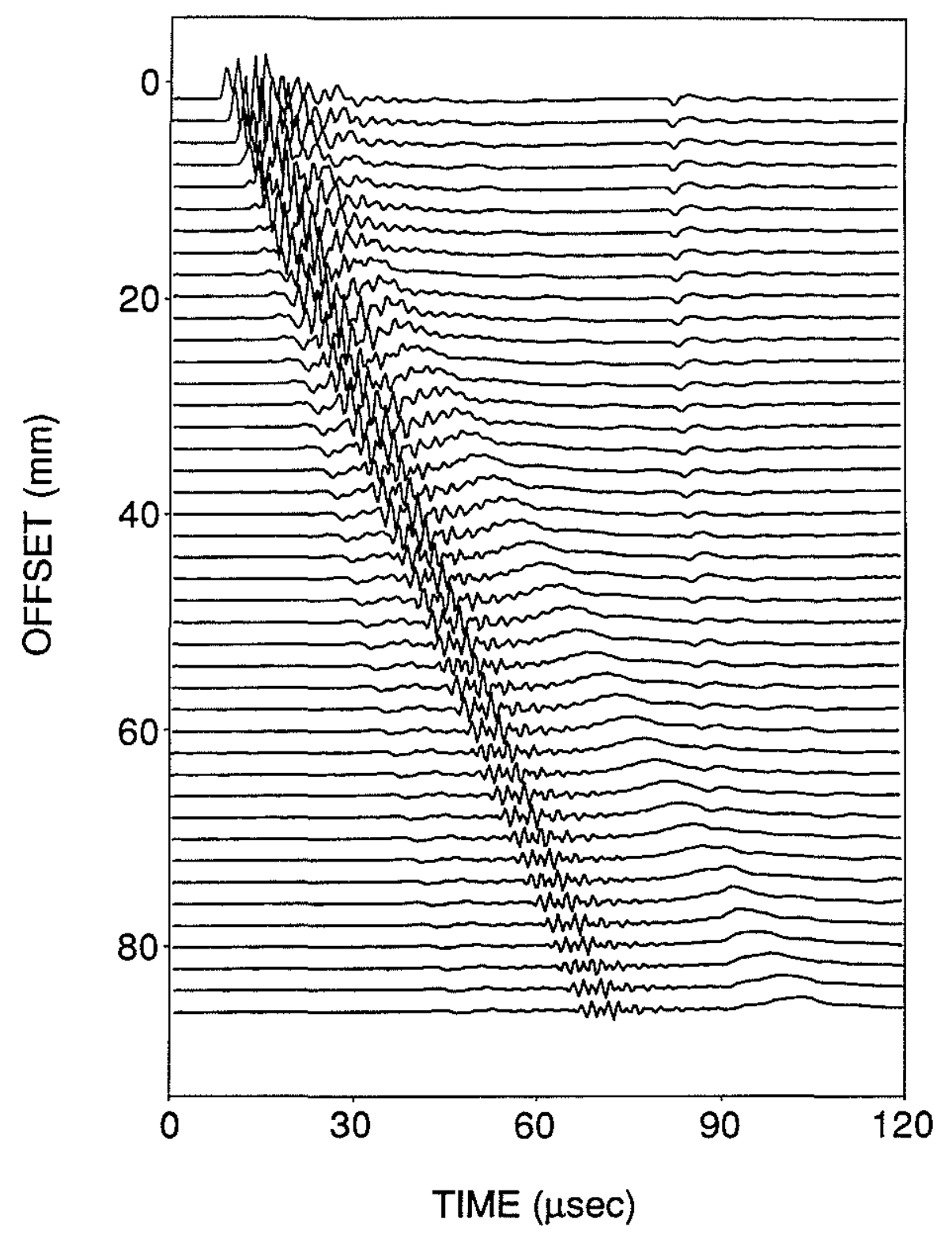

Figure 6: Laboratory data for which lucite was used to mimic a slow formation. 

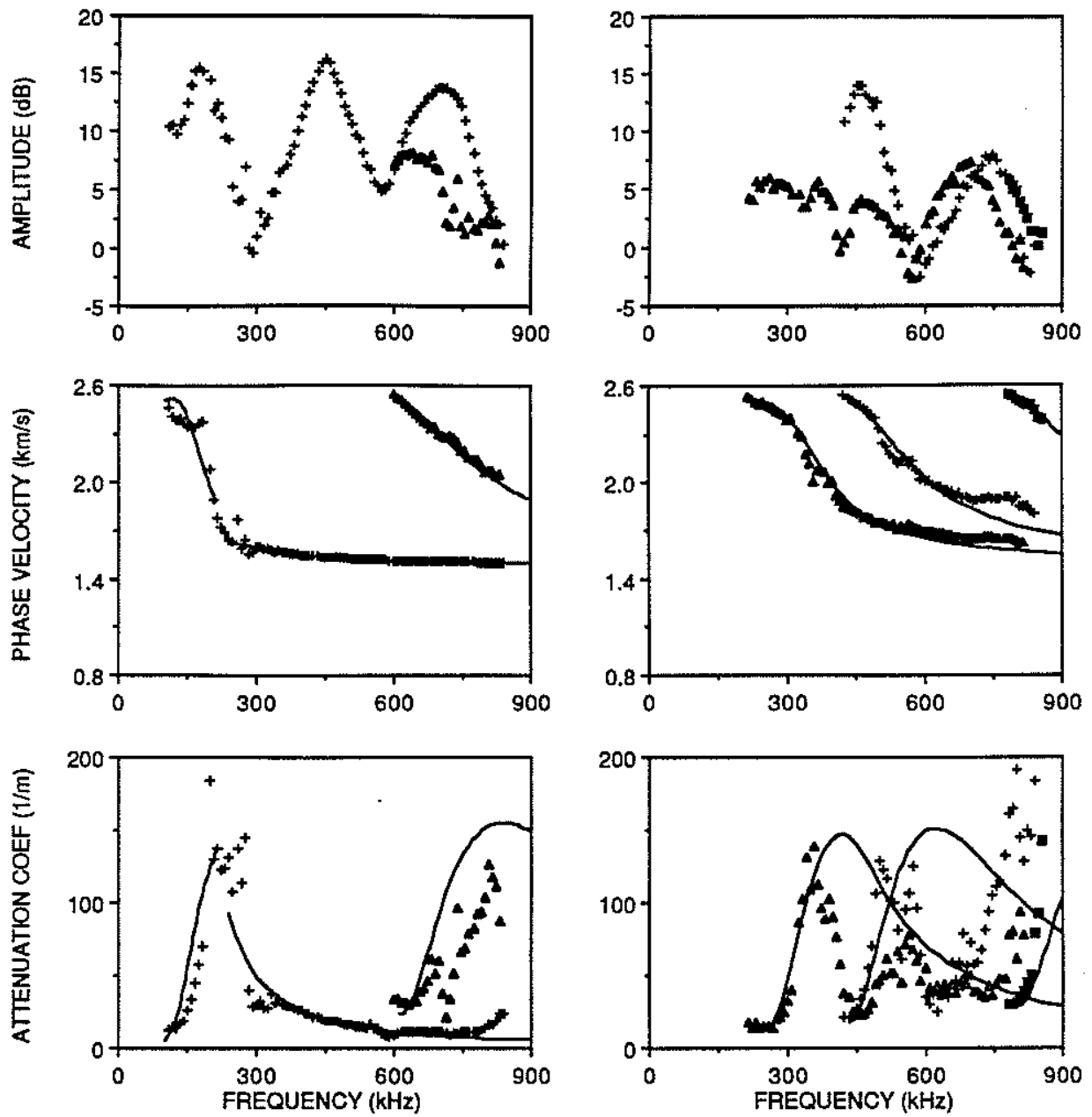

Figure 7: Amplitude, phase velocity, and attenuation estimates from the laboratory data. These estimates are plotted on different graphs for clarity, and amplitude is scaled to decibels. The solid lines are theoretical predictions based upon measured values for the velocity and attenuation. (a), (b), and (c) Results for first and fourth leaky-P modes (plus signs and solid triangles, respectively). (d), (e), and (f) Results for second, third, and fifth leaky-P modes (open triangles, plus signs, and solid squares, respectively). (g), (h), and (i) Results for the tube wave (open squares). 

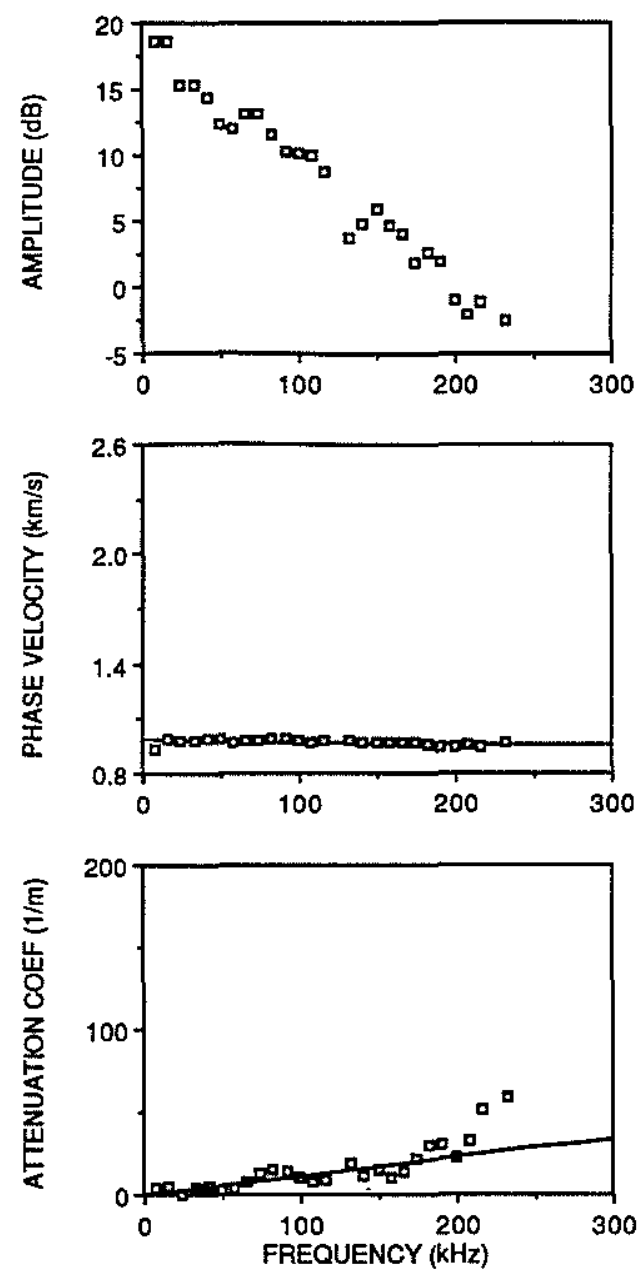


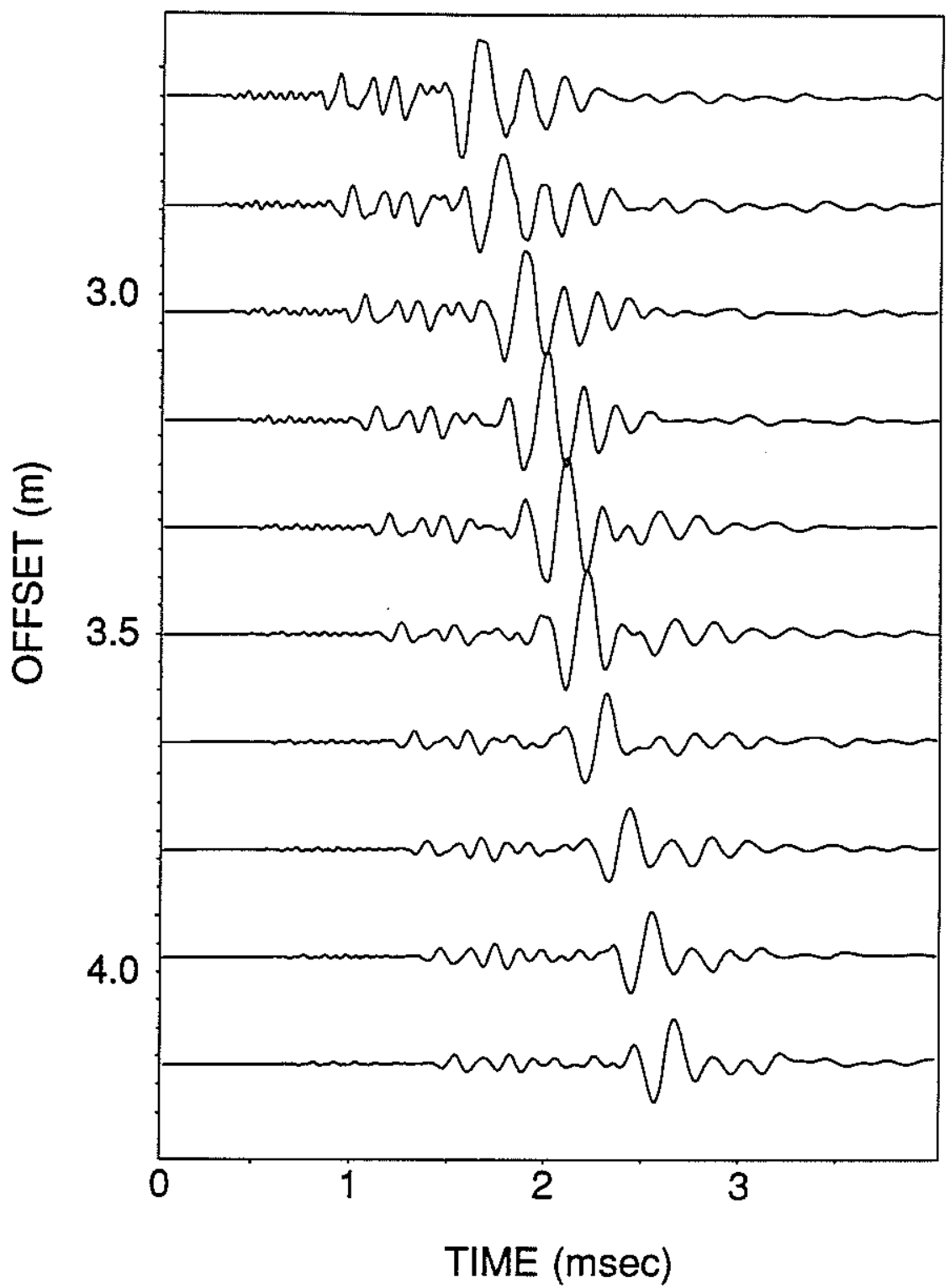

Figure 8: Field data collected in a highly permeable sandstone. 

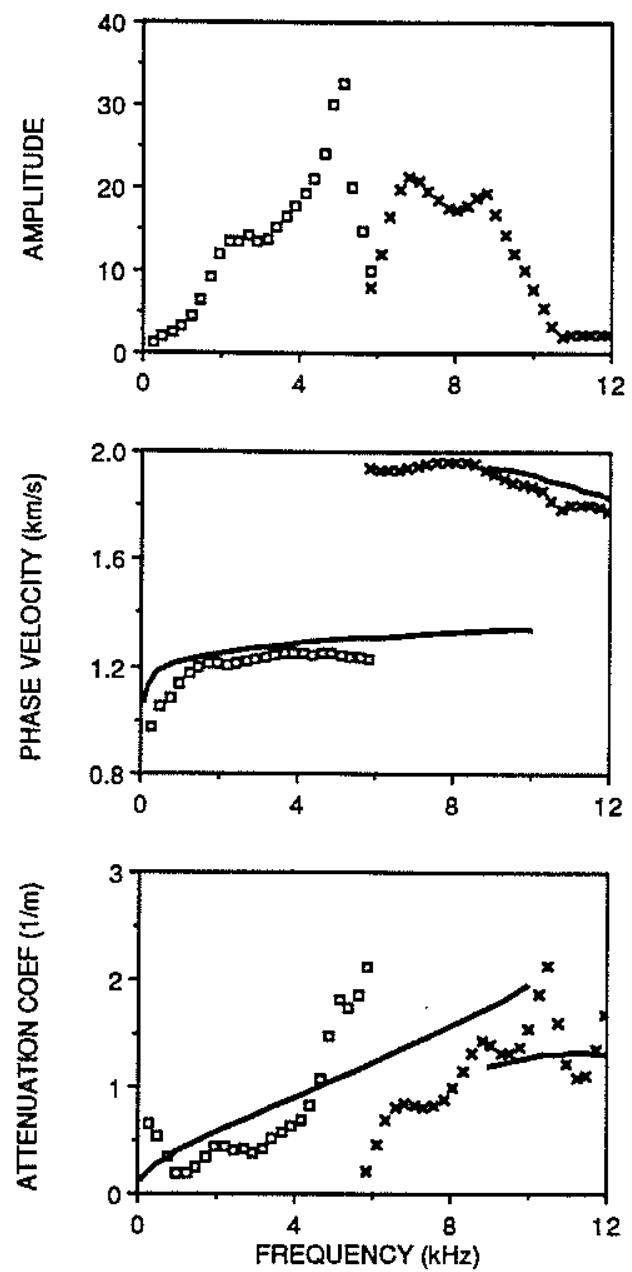

Figure 9: Amplitude (a), phase velocity (b), and attenuation (c) estimates from the field data. The solid lines are predictions constrained by the $\mathrm{P}$ - and $\mathrm{S}$-wave velocities and other well log measurements. 